\title{
Characterization of tunable magnetic sensor using bias magnetic field of a hard magnetic film magnetized by pulsed-magnetic field
}

\author{
Makoto Sonehara $^{1}$, Member, IEEE, Hiroki Kamada ${ }^{1}$, Satoko Iida ${ }^{2,1}$, and Toshiro Sato ${ }^{1}$, Member, IEEE \\ ${ }^{1}$ Spin Device Technology Center, Shinshu University, 4-17-1 Wakasato, Nagano 380-8553, Japan \\ ${ }^{2}$ Kisarazu National College of Technology, 2-11-1 Kiyomidaihigashi, Kisarazu, Japan
}

\begin{abstract}
A tunable MR sensor with variable sensitivity and measuring range is fabricated and characterized. The sensor has been fabricated using a combination of Ni-Fe soft magnetic film, Fe-Co-Sm hard magnetic film. The sensor is placed in a magnetizing coil for the process of magnetizing the hard magnetic film. The sensitivity and the measuring range of the sensor are tuned by bias magnetic field produced by the magnetic poles of the hard magnetic film. The sensitivity change was about $60 \%$, and the measuring range change was about $30 \%$ in the tunable MR sensor from the experimental results. The bias magnetic field is controlled by changing amplitude of the pulsed magnetic field in the magnetizing for the hard magnetic film. The control power for one time tuning is about $18 \mu \mathrm{Wh}$.
\end{abstract}

Index Terms - Tunable magnetic sensor, variable sensitivity and measuring range, hard magnetic film, soft magnetic film, pulsed magnetic field magnetizing method.

\section{INTRODUCTION}

$\mathrm{T}$ he magnetic sensors with the soft magnetic film such as magnetoresistance (MR) and giant magnetoresistance (GMR) sensors are used for the current sensors, electric compasses, magnetic heads, etc [1]-[3]. Recently, an optical probe current sensor for electric vehicles (EVs) and the hybrid electric vehicles (HEVs) using the Kerr effect of a soft magnetic film was fabricated and characterized [4],[5]. Its advantage is high noise immunity and electrical insulation. The magnetic sensors with high sensitivity and wide measuring range are required for EVs/ HEVs application. For the safety, wide measuring range is required at high load current of electric motor such as sudden acceleration, and high sensitivity is required at low load current such as low speed driving in a traffic jam. However, there is a reciprocal relationship between the sensitivity and the measuring range of the magnetic sensors. Both the sensitivity and the measuring range dependents on the internal energy which include the anisotropy energy of the soft magnetic film [2]. It is difficult to control the internal energy of the sensor using the soft magnetic film only.

Thus a method to apply external energy to the soft magnetic film has been suggested. For example, B. K. Kuanr et al. reported a tunable FMR (ferromagnetic resonance) filter using bias magnetic field applied in easy magnetization direction of the magnetic film [6]. The control power for changing FMR frequency becomes large in case of using dc current for generating bias magnetic field. Moreover, the dc current for the external magnetic field energy (the Zeeman energy) must be kept a constant value in order to maintain the fixed FMR frequency.

In this study, a hard magnetic film based magnetic field biasing the tunable magnetic sensor was proposed. The magnetic sensor has a combination of the soft and hard

Manuscript received November 2, 2012; revised January 19, 2013. Current version published Xxxxx XX, 2013. Corresponding author: Makoto Sonehara (e-mail: makoto@shinshu-u.ac.jp; optional phone: +81-26-269-5198; optional fax: +81-26-269-5215).

Digital Object Identifier 10.1109/TMAG.2013.xxxxxxx magnetic combination films. Therefore the sensitivity and the measuring range in the magnetic sensor are tuned by the Zeeman energy from bias magnetic field in hard magnetic film.

This paper describes a development and characterizations of the tunable MR sensor with Ni-Fe soft magnetic film and FeCo-Sm hard magnetic film as fundamental experiment of the optical probe current sensor.

\section{BASIC PRINCIPLE OF TUNABLE SENSOR}

\section{A. Tunable method of bias magnetic field by hard magnetic} film in soft/hard magnetic films

Figure 1, 2 and 3 show a basic principle of the internal energy control for the soft magnetic film using a bias magnetic field of the hard magnetic film. Figure 1 shows a schematic explanation of tunable MR sensor with soft/hard magnetic combination films and applying bias magnetic field from the hard magnetic film. As shown in Fig. 2, the hard magnetic film is magnetized by the pulsed magnetic field magnetizing method. The first pulsed-current $I_{\mathrm{p} 1}$ for saturating magnetization has constant amplitude in order to erase the hysteresis of the magnetization. The second pulsed-current $I_{\mathrm{p} 2}$ has variable amplitude to change the remanent magnetization $M_{\mathrm{r}}$ of the hard magnetic film. The range of the remanent

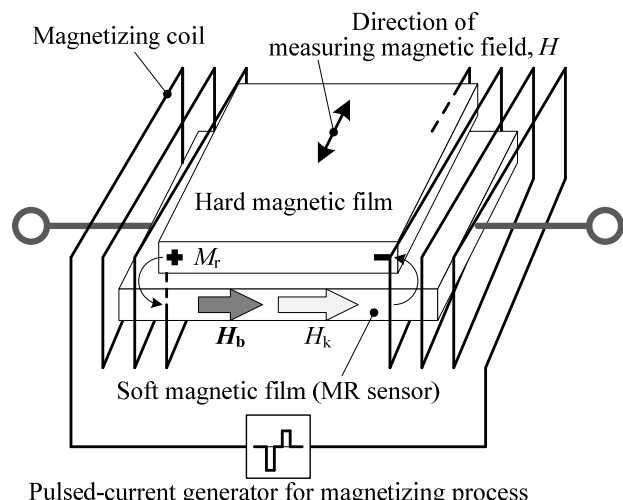

Fig. 1. Schematic explanation of tunable MR sensor with soft/hard magnetic combination films and applying bias magnetic field from hard magnetic film. 


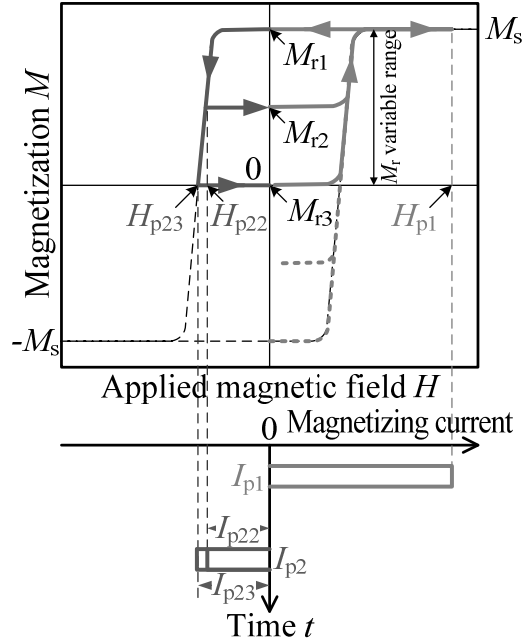

Fig. 2. Schematic hysteresis curve with varying remanent magnetization $M_{\mathrm{r}}$ in hard magnetic film using a pulsed magnetic field magnetizing method in the tunable sensor.

Direction of measuring magnetic field, $H$

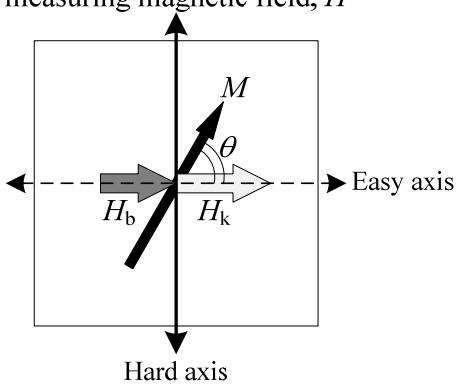

Fig. 3. Schematic top view of relation between the magnetization $M$, the uniaxial anisotropy field $H_{\mathrm{k}}$, the bias magnetic field $H_{\mathrm{b}}$, the measuring magnetic field $H$, and the angle $\theta$ in soft magnetic film.

magnetization $M_{\mathrm{r}}$ is approximately $-M_{\mathrm{s}}$ to $M_{\mathrm{s}}$, when the hard magnetic film has high squareness ratio. In case of $I_{\mathrm{p} 2}=0, M_{\mathrm{r}}$ and $H_{\mathrm{b}}$ are maximum. When decreasing $I_{\mathrm{p} 2}$ as shown $I_{\mathrm{p} 22}$ in the Fig. 2, $M_{\mathrm{r}}$ and $H_{\mathrm{b}}$ are decreased. In case of $I_{\mathrm{p} 2}=I_{\mathrm{p} 23}, M_{\mathrm{r}}$ and $H_{\mathrm{b}}$ are 0 . On the other hand, a magnetic pole is reversed in $I_{\mathrm{p} 2}<$ $I_{\mathrm{p} 23}$, namely, the relation between $M_{\mathrm{r}}$ or $H_{\mathrm{b}}$ and $I_{\mathrm{p} 2}-I_{\mathrm{p} 23}$ are expressed to an even function.

The bias magnetic field $H_{\mathrm{b}}$ applied in easy axis of the soft magnetic film can be controlled by changing $M_{\mathrm{r}}$, and then the internal energy $E$ of the soft magnetic film can be tuned as shown Eq. (1);

$$
E=M_{\mathrm{s}}\left(\frac{H_{\mathrm{k}}}{2} \sin ^{2} \theta-H_{\mathrm{b}} \cos \theta-H \sin \theta\right)
$$

where $M_{\mathrm{s}}$ is the saturation magnetization, and $H_{\mathrm{k}}$ is the uniaxial anisotropy field in the soft magnetic film, $\theta$ is the angle of magnetization $\boldsymbol{M}$ from the easy axis in the soft magnetic film as shown in Fig. 3, and $H$ is the measuring magnetic field.

The angle $\theta$ is decided by Eq. (1) and (2):

$$
\frac{\partial}{\partial \theta} E=0, \quad \frac{\partial^{2}}{\partial \theta^{2}} E>0
$$

\section{B. Tunable MR sensor}

Figure 4 shows a relation between an absolute angle $|\theta|$ and $|H| / H_{\mathrm{k}}$. When the bias magnetic field $H_{\mathrm{b}}$ is 0 , the absolute angle $|\theta|$ equals to $90^{\circ}$ in $|H| \geq H_{\mathrm{k}}$. The output of the MR sensor is dependent on the angle $\theta$ as following Eq. (3), and the output of the optical probe current sensor is dependent on the angle $\theta$, too [4], [5]. Therefore the measuring range of the sensor is limited in $|H|=H_{\mathrm{k}}$ in this case.

$$
\rho=\rho_{0}+\Delta \rho \cos ^{2} \theta
$$

On the other hand, when the bias magnetic field $H_{\mathrm{b}}$ is applied, the measuring range of the sensor is unlimited. The sensitivity of the sensor is decreased when increasing the bias magnetic field $H_{\mathrm{b}}$.

\section{FABRICATION OF SOFT/HARD MAGNETIC FILMS}

Figure 5 shows a schematic and dimension of the fabricated tunable MR sensor using bias magnetic field of the hard magnetic film magnetized by the pulsed magnetic field magnetizing method. First, a photo resist pattern with $50 \mu \mathrm{m}$ width air-gap like as lattice pattern and $200 \mu \mathrm{m}$ length segment for hard magnetic film was formed on a glass substrate in order to improve the spatial distribution of bias magnetic field in the soft magnetic film [7]. Then $1 \mu \mathrm{m}$ thick $\mathrm{Fe}_{56.7} \mathrm{Co}_{36.7} \mathrm{Sm}_{6.6}$ (at.\%) amorphous hard magnetic film with 1.4 $\mathrm{T}$ of $M_{\mathrm{s}}$, and then $50 \mathrm{~nm}$ thick $\mathrm{SiO}_{2}$ film for anti-oxidation barrier layer were deposited by r.f. magnetron sputtering. Then the photo resist was removed by lift-off method. Second, 50 nm thick $\mathrm{Ni}_{78} \mathrm{Fe}_{22}$ (at.\%) soft magnetic film for the MR sensor

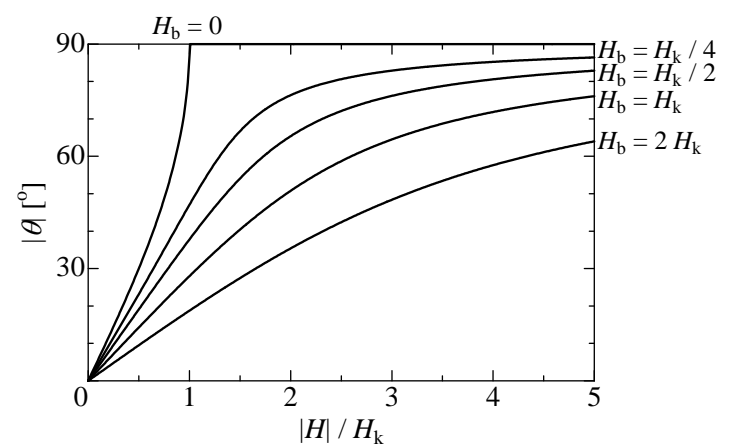

Fig. 4. Relation between the absolute angle $|\theta|$ and $|H| / H_{\mathrm{k}}$ in an uniaxial anisotropy magnetic film with the bias magnetic field.

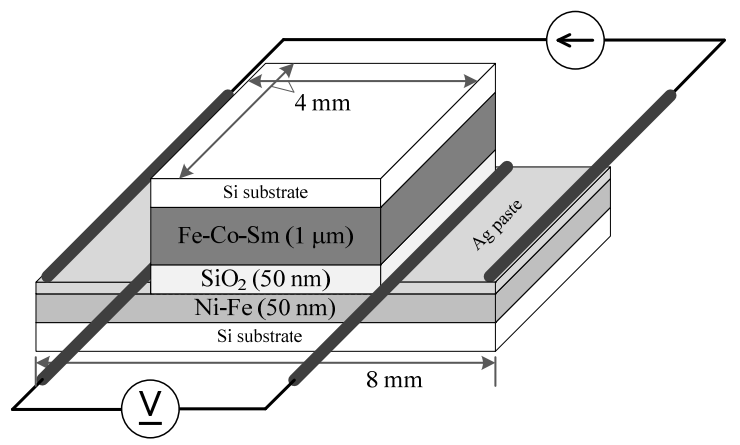

Fig. 5. Schematic and dimension of fabricated tunable MR sensor with $\mathrm{Ni}-\mathrm{Fe}$ soft/Fe-Co-Sm hard magnetic combination films. 
with $3.2 \mathrm{kA} / \mathrm{m}$ of $H_{\mathrm{k}}$ and $1 \%$ of MR ratio was deposited by r.f. magnetron sputtering on another glass substrate. Third, the $\mathrm{SiO}_{2} / \mathrm{Fe}-\mathrm{Co}-\mathrm{Sm}$ hard magnetic film and the Ni-Fe soft magnetic film were diced to $4 \mathrm{~mm}$ square size and $4 \mathrm{~mm} \times 8$ $\mathrm{mm}$ size respectively by using a dicer. Finally, two films were joined, and then $\mathrm{Cu}$ wires for the four-terminal method were connected by using Ag paste on the soft magnetic film as shown Fig. 5 [8].

\section{MEASUREMENT}

The tunable MR sensor was put in the center of a magnetizing solenoid coil with $18 \mathrm{~mm}$ of length, $10 \mathrm{~mm}$ of mean diameter, and 568 turns as shown Fig. 1. The magnetizing solenoid coil connected to a handmade pulse current generator. A relation between the magnetizing field $H_{\mathrm{p}}$ and the pulsed-current $I_{\mathrm{p}}$ from the dc supply is following equation; $H_{\mathrm{p}}=7.14 I_{\mathrm{p}}[\mathrm{kA} / \mathrm{m}]$. The first pulsed-current with constant amplitude $I_{\mathrm{p} 1}$ was kept to $7.5 \mathrm{~A}\left(H_{\mathrm{p} 1}=53.5[\mathrm{kA} / \mathrm{m}]\right)$ in order to saturate the Fe-Co-Sm hard magnetic film. On the other hand, the second pulsed-current with variable amplitude $I_{\mathrm{p} 2}$ were established $-6.0 \leq I_{\mathrm{p} 2} \leq 0$ [A] (i.e. $-43 \leq H_{\mathrm{p} 2} \leq 0$ $[\mathrm{kA} / \mathrm{m}])$. The current pulse-width was about $1 \mathrm{~ms}$ in this experiment.

The sensor and the magnetizing coil were put in a solenoid coil with $95 \mathrm{~mm}$ of length, $103 \mathrm{~mm}$ of mean diameter, and 1150 turns for the applied magnetic field $H$ which connected to a dc supply (KENWOOD; PR18-1.2A). A relation between the applied magnetic field $H$ and the current $I$ from the dc supply is following equation; $H=8.16 I[\mathrm{kA} / \mathrm{m}]$.

A variation of a resistivity in the tunable MR sensor with the applied magnetic field $H$ was measured by four-terminal method using a digital multi meter (ADVANTEST; R6871EDC).

\section{RESUlTS AND DISCUSSION}

A. Relation between remanent magnetization and pulsedcurrent in $\mathrm{Ni}-\mathrm{Fe} / \mathrm{Fe}-\mathrm{Co}-\mathrm{Sm}$ films for tunable sensor

Figure 6 shows the relation between the remanent magnetization $M_{\mathrm{r}}$ in the Fe-Co-Sm hard magnetic film, the second pulsed-current with variable amplitude $I_{\mathrm{p} 2}$, and the variable magnetizing field $H_{\mathrm{p} 2}$ in the Ni-Fe/Fe-Co-Sm

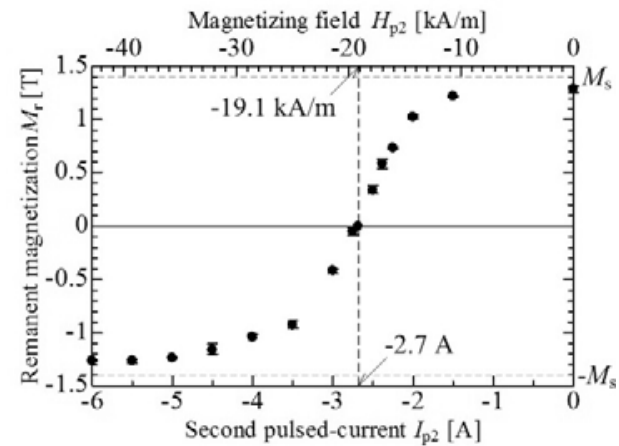

Fig. 6. Relation between the remanent magnetization $M_{\mathrm{r}}$ in Fe-Co-Sm hard magnetic film, the second pulsed-current with variable amplitude $I_{\mathrm{p} 2}$, and the variable magnetizing field $H_{\mathrm{p} 2}$ in Ni-Fe/Fe-Co-Sm combination films for the tunable MR sensor. combination films of the tunable MR sensor. The remanent magnetization $M_{\mathrm{r}}$ was measured by using a vibrating sample magnetometer (VSM, Riken Denshi; BHV-55). From Fig. 6, the remanent magnetization $M_{\mathrm{r}}$ is equal to $0[\mathrm{~T}]$ at $I_{\mathrm{p} 2}=-2.7$ [A] $\left(H_{\mathrm{p} 2}=-19.1[\mathrm{kA} / \mathrm{m}]\right)$, so then bias magnetic field $H_{\mathrm{b}}=0$ is expected. $M_{\mathrm{r}}$ increases when $I_{\mathrm{p} 2}$ increases. $M_{\mathrm{r}}$ is approximately saturated in $I_{\mathrm{p} 2}>-1.3$ [A]. On the other hand, $M_{\mathrm{r}}$ deceases when $I_{\mathrm{p} 2}$ decreases. $M_{\mathrm{r}}$ is approximately saturated in $I_{\mathrm{p} 2}<-5$ [A]. These results follow by the Section II A and Fig. 2.

\section{B. Relation between a change in resistivity and applied magnetic field in tunable MR sensor with $\mathrm{Ni}-\mathrm{Fe} / \mathrm{Fe}-\mathrm{Co}-\mathrm{Sm}$ combination films}

Figure 7 shows the relation between a change in resistivity $\Delta \rho / \rho$, the applied magnetic field $H$, and the typical variable magnetizing field $H_{\mathrm{p} 2}$ in the tunable MR sensor. Furthermore the relation between a change in resistivity $\Delta \rho / \rho$ and the applied magnetic field $H$ of a MR sensor without hard magnetic film was described in Fig. 7. In addition, the calculated results were estimated by Eq. (1)-(3). The bias magnetic field $H_{\text {be }}$ used for the calculation were fitted by the measured results.

From Fig. 7, the sensitivity decreases, and the measuring range increases when $H_{\mathrm{p} 2}$ increases. The calculated results agree well with the measurements in the low field $H$ region $(H$ $<3[\mathrm{kA} / \mathrm{m}])$. However, the calculated results are inconsistent with the measurements in the vicinity of saturating $\Delta \rho / \rho$. The authors considered that the main reason is an effect of anisotropy dispersion in the soft magnetic film.

The measured result at $H_{\mathrm{p} 2}=-19.1[\mathrm{kA} / \mathrm{m}]$ is inconsistent with that of without hard magnetic film, and the bias magnetic field $H_{\text {be }}$ was estimated about $0.7 \mathrm{kA} / \mathrm{m}$ from the results. The reason is currently not clear. However, the authors observed many stripe magnetic domains in the hard magnetic film at $H_{\mathrm{p} 2}$ $=-19.1[\mathrm{kA} / \mathrm{m}]$. Therefore it is expected that $0.7 \mathrm{kA} / \mathrm{m}$ of microscopic bias magnetic field was occurred by the magnetic poles in the magnetic domains. The authors examine and research a mean of $H_{\text {be }}=0$.

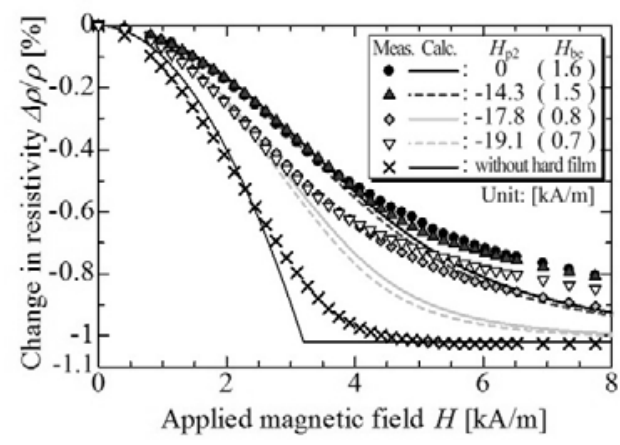

Fig. 7. Relation between change in resistivity $\Delta \rho / \rho$, applied magnetic field $H$, and typical variable magnetizing field $H_{\mathrm{p} 2}$ from hard magnetic film in the tunable MR sensor. 


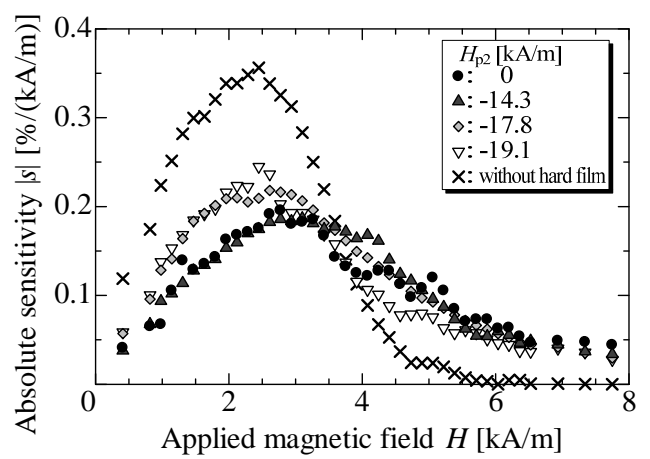

Fig. 8. Relation between change in absolute sensitivity $|s|$, applied magnetic field $H$, and variable magnetizing field $H_{\mathrm{p} 2}$ from hard magnetic film in the tunable MR sensor.

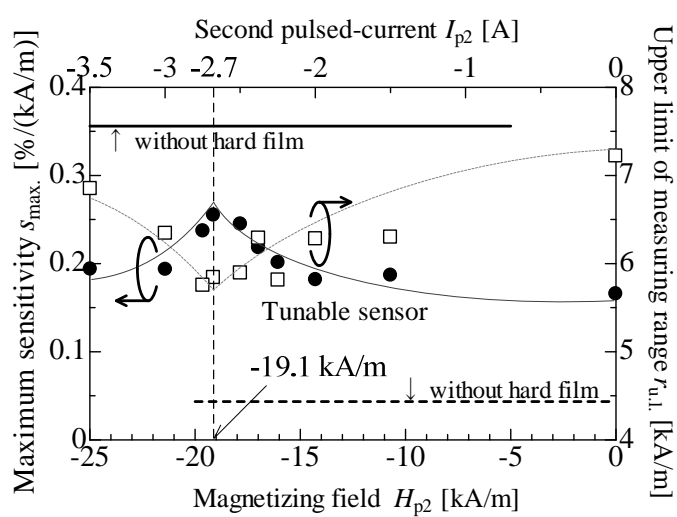

Fig. 9. Relation between maximum sensitivity $s_{\max }$, upper limit of measuring range $r_{\mathrm{u} . \mathrm{l}}$, variable magnetizing field $H_{\mathrm{p} 2}$ from hard magnetic film, and second pulsed-current $I_{\mathrm{p} 2}$ in the tunable MR sensor.

\section{Variable sensitivity and measuring range in tunable $M R$} sensor with $\mathrm{Ni}-\mathrm{Fe} / \mathrm{Fe}-\mathrm{Co}-\mathrm{Sm}$ combination films

Figure 8 shows the relation between an absolute sensitivity $|s|$, the applied magnetic field $H$, and the typical variable magnetizing field $H_{\mathrm{p} 2}$ from hard magnetic film in the tunable MR sensor. The absolute sensitivity $|s|[\% /(\mathrm{A} / \mathrm{m})]$ was defined by $|\mathrm{d}(\Delta \rho / \rho) / \mathrm{d} H|$ and calculated from Fig. 7 .

Figure 9 shows the relation between a maximum sensitivity $s_{\text {max. }}$ in each data, an upper limit of measuring range $r_{\text {u.l. }}$, the variable magnetizing field $H_{\mathrm{p} 2}$, and the second pulsed-current $I_{\mathrm{p} 2}$ in the tunable MR sensor. The upper limit of measuring range $r_{\text {u.l. }}$ is defined at $|s|=0.05[\% /(\mathrm{kA} / \mathrm{m})]$ in Fig. 8 .

From Fig. 8 and 9 , the sensitivity $s$ decreases when $H_{\mathrm{p} 2}$ increases. The sensitivity in the tunable MR sensor was tuned about $60 \%$. If some problems such as the anisotropy dispersion in soft magnetic film were solved, the result of the tunable MR sensor with $M_{\mathrm{r}}=0$ will correspond to that of the sensor without hard magnetic film, so then a change in sensitivity is expected about $110 \%$.

On the other hand, from Fig. 9, the upper limit of measuring range $r_{\text {u.l. }}$ increases when $H_{\mathrm{p} 2}$ increases. $r_{\text {u.l. }}$ in the tunable MR sensor was tuned about $30 \%$. A change in $r_{\text {u.l. }}$ is expected about $60 \%$ for the reason mentioned above.

\section{CONCLUSIONS}

This paper describes a development and characterizations of the tunable MR sensor using a bias magnetic field of the hard magnetic film magnetized by pulsed magnetic field magnetizing method. The results obtained are as follows:

(1) The remanent magnetization $M_{\mathrm{r}}$ of the Ni-Fe/Fe-Co-Sm magnetic combination films in the tunable MR sensor is changed from -1.3 to 1.3 [T] by variable bias magnetic field of the Fe-Co-Sm hard magnetic film. $M_{\mathrm{r}}$ is equal to 0 [T] at $I_{\mathrm{p} 2}=-2.7[\mathrm{~A}]\left(H_{\mathrm{p} 2}=-19.1[\mathrm{kA} / \mathrm{m}]\right)$.

(2) The sensitivity decreased, and the measuring range increased when increasing $H_{\mathrm{p} 2}$ in the tunable MR sensor with the $\mathrm{Ni}-\mathrm{Fe} / \mathrm{Fe}-\mathrm{Co}-\mathrm{Sm}$ magnetic combination films.

(3) The sensitivity change was about $60 \%$, and the measuring range change was $30 \%$ in the tunable MR sensor. The potential variable values of the two parameters were expected about $110 \%$ and $60 \%$, respectively.

Thus the tunable sensor using a bias magnetic field of a hard magnetic film magnetized by the pulsed magnetic field magnetizing method is demonstrated as a part of fundamental experiment of the optical probe current sensor.

\section{ACKNOWLEDGMENT}

The corresponding author really thank to Dr. Kenji Ikeda, R\&D Center, Taiyo Yuden Co., Ltd. for assisting the fabrication of Fe-Co-Sm hard magnetic film.

A part of this research was supported by "Adaptable and Seamless Technology Transfer Program (A-STEP; AS242Z02104J), Japan Science and Technology Agency”.

\section{REFERENCES}

[1] J. S. Y. Feng, L. T. Romankiw, and D. A. Thompson, "Magnetic selfbias in the barber pole MR structure”, IEEE Trans. Magn., Vol.13, No.5, pp.1466-1468 (1977)

[2] T. Yeh, and W. F. Witcraft, "Effect of Magnetic Anisotropy on Signal and Noise of NiFe Magnetoresistive Sensor", IEEE Trans. Magn., Vol.31, No.6, pp.3131-3133 (1995).

[3] J. M. Daughton, and Y. J. Chen, "GMR Materials for Low Field Applications”, IEEE Trans. Magn., Vol.29, No.6, pp.2705-2710 (1993).

[4] M. Sonehara, K. Asanuma, N. Otani, T. Goto, Y. Kikuchi, T. Sato, K. Yamasawa, and Y. Miura, "Fundamental Study of Optical Probe Current Sensor using Kerr Effect of Single Magnetic Domain Film”, Proc. of the 18th IEEE Conf. on Sensors (IEEE Sensors 2009 Conf.), pp.1232-1237 (2009).

[5] K. Ogawa, S. Suzuki, M. Sonehara, T. Sato, and K. Asanuma, “Optical Probe Current Sensor Module using the Kerr Effect of Exchangecoupled Magnetic Film and its Application to IGBT Switching Current Measurements”, International Jour. on Smart Sensing and Intelligent Systems, Vol.5, No.2, pp.347-361 (2012).

[6] B. K. Kuanr, V. Veerakumar, K. Lingam, S.R. Mishra, Alka V.Kuanr, R.E. Camley, and Z. Celinski, "Microstrip-Tunable Band-Pass Filter Using Ferrite (Nanoparticles) Coupled Lines”, IEEE Trans. Magn., Vol.45, No.10, pp.4226-4229 (2009).

[7] M. Yuki, M. Sonehara, T. Sato, and K. Ikeda "Fundamental Study on Tunable RF Magnetic Devices Using DC Bias Magnetic Field Generated by Permanent Magnet Film”, J. Magn. Soc. Jpn., Vol.36, No.3, pp.229234 (2012). (in Japanese)

[8] M. Sonehara, T. Shinohara, T. Sato, K. Yamasawa, and Y. Miura, "Strain sensor using stress-magnetoresistance effect of $\mathrm{Ni}-\mathrm{Fe} / \mathrm{Mn}-\mathrm{Ir}$ exchange-coupled magnetic film”, Journal of Applied Physics, Vol.107, No.9, pp.09E718-1-09E718-3 (2010). 